\title{
Cardiac magnetic resonance imaging for the assessment of ventricular function, geometry, and viability before and after surgical ventricular reconstruction
}

\author{
Jan Hüther, ${ }^{\mathrm{a}}$ Torsten Doenst, MD, PhD, ${ }^{\mathrm{a}, \mathrm{d}}$ Stefan Nitzsche, MD,${ }^{\mathrm{b}}$ Holger Thiele, MD, PhD, ${ }^{\mathrm{c}}$
} Friedrich-Wilhelm Mohr, MD, PhD, ${ }^{\mathrm{a}}$ and Matthias Gutberlet, $\mathrm{MD}, \mathrm{PhD}^{\mathrm{b}}$

\begin{abstract}
Objective: Surgical ventricular reconstruction is a treatment option for patients with apical akinesia or dyskinesia. The Surgical Treatment for Ischemic Heart Failure trial recently demonstrated its safety but no added benefit to bypass surgery, although the trial's inclusion criteria did not contain shape or viability parameters. However, we evaluated cardiac magnetic resonance-derived parameters as potential predictors of function after surgical ventricular reconstruction.
\end{abstract}

Methods: In 24 patients with cardiac magnetic resonance before and after surgical ventricular reconstruction, we assessed cardiac volumes, function, scar, and geometry (sphericity index, short to long axis; apical conicity index, apical to short axis; apical volume index, apical to basal volume).

Results: Surgical ventricular reconstruction significantly reduced ventricular volumes $(-64.2 \%)$ and increased global ejection fraction by $12 \%(P<.01)$. The sphericity index was increased by surgical ventricular reconstruction $(0.60 \pm 0.07$ vs. $0.76 \pm 0.13 . P<.05)$ indicative of ball shapes. The apical to short axis $(0.71 \pm 0.13$ to 0.58 $\pm 0.09)$ and apical to basal volume $(0.45 \pm 0.08$ to $0.26 \pm 0.11)$ decreased, consistent with aneurysm removal. The preoperative ventricles contained $25 \% \pm 14 \%$ of scar (apical: $72 \% \pm 8 \%$, midcavity: $38 \% \pm 14 \%$, basal region: $10 \% \pm 12 \%$ ). Patients with ejection fraction improvement greater than $12 \%$ had less basal scar preoperatively and showed greater apical to basal volume reduction than those with ejection fraction improvement less than $12 \%$. Basal wall motion scores did not differ between the subgroups. However, multivariable analysis identified only ejection fraction and urgency of operation as independent risk predictors.

Conclusions: The assessment of basal viability and the determination of the apical to basal volume may allow identifying the subgroup of patients who potentially derive a benefit from surgical ventricular reconstruction. A larger study is needed to support this conclusion. (J Thorac Cardiovasc Surg 2011;142:1515-22)

\section{Supplemental material is available online.}

Cardiac magnetic resonance (CMR) is often used to assess ventricular shape, volume, and viability before a revascularization or ventricular reconstruction procedure. ${ }^{1,2}$ Surgical

\footnotetext{
From the Departments of Cardiac Surgery, ${ }^{\mathrm{a}}$ Diagnostic and Interventional Radiology, ${ }^{\mathrm{b}}$ and Cardiology, ${ }^{\mathrm{c}}$ University of Leipzig Heart Center Leipzig, Germany; and Department of Cardiothoracic Surgery, ${ }^{\mathrm{d}}$ University of Jena, Jena, Germany.

T.D. was Heisenberg-Professor of the Deutsche Forschungsgemeinschaft (DFG) at the University of Leipzig until August 2010 and was supported by grants from the DFG (Do602/4-1, 6-1, 8-1, 9-1 and Wa 2433/2-1).

Disclosures: Authors have nothing to disclose with regard to commercial support.

J.H. and T.D. contributed equally to this article.

Received for publication Oct 26, 2010; revisions received April 3, 2011; accepted for publication April 26, 2011; available ahead of print Spet 12, 2011.

Address for reprints: Torsten Doenst, MD, PhD, Department of Cardiothoracic Surgery, University of Jena, Erlanger Allee 101, 07747 Jena, Germany (E-mail: doenst@med.uni-jena.de).

$0022-5223 / \$ 36.00$

Copyright (c) 2011 by The American Association for Thoracic Surgery

doi:10.1016/j.jtcvs.2011.04.040
}

ventricular reconstruction (SVR) has been an accepted treatment option for patients with ischemic heart failure presenting with postinfarct akinesia or dyskinesia. ${ }^{3}$ These patients usually have large aneurysms giving the typical dyskinetic picture of left ventricular apex, but SVR has also been advocated for patients with dilated hearts and akinetic anterior walls. ${ }^{3}$ The Surgical Treatment for Ischemic Heart Failure (STICH) trial just addressed the impact of SVR on survival and reported that SVR was safe, but did not add an extra survival or quality of life benefit to the effects of bypass surgery alone. ${ }^{4}$ Although this outcome is definitive and certainly represents a setback for the broad application of the procedure, the trial's inclusion criteria for patients with anterior akinesia or dyskinesia did not include parameters determining shape, regional contractility, or myocardial viability. Thus, the demonstration of the safety of the procedure may therefore be used as an encouragement to clearly define the subgroup of this patient population who does derive a benefit.

To achieve this goal, new, regional parameters and the degree of viable myocardium need to be assessed. The commonly used parameters to address the effectiveness of the 


$$
\begin{aligned}
& \text { Abbreviations and Acronyms } \\
& \text { ACI }=\text { apical conicity index } \\
& \text { AVI }=\text { apical volume index } \\
& \text { CMR }=\text { cardiac magnetic resonance } \\
& \text { EF }=\text { ejection fraction } \\
& \text { LAL }=\text { long-axis length } \\
& \text { SAL }=\text { short-axis length } \\
& \text { SpI } \text { sphericity index } \\
& \text { STICH }=\text { Surgical Treatment for Ischemic Heart } \\
& \text { Failure } \\
& \text { SVR }=\text { surgical ventricular reconstruction }
\end{aligned}
$$

procedure have been the left ventricular ejection fraction (EF) and left ventricular volumes, but both are changed as a natural consequence of this volume-altering procedure. Shape indices obtained by conventional ultrasound ${ }^{5}$ or 3-dimensional echocardiography ${ }^{6}$ have been introduced and are tools to describe regional and global left ventricular shape. However, the availability of echocardiographic data pre- and post-SVR are still limited, and those of CMR are practically absent.

We therefore analyzed all patients in our database who obtained CMR before and after SVR and assessed myocardial function, geometry, and viability pre- and post-SVR.

\section{MATERIAL AND METHODS \\ Patients}

In this retrospective analysis, we identified 24 patients in our database between 2002 and 2008 who underwent SVR and obtained CMR both before and after the procedure (192 patients underwent SVR in this time period). These patients were used for the present analysis. All patients agreed to the scientific assessment of their parameters by signing a consent form preoperatively. The management of our database is consistent with the qualifications set forth by the ethics committee of the University of Leipzig. The conduct of the study is in agreement with the Declaration of Helsinki.

\section{Conduct of Operation and Perioperative Care}

The procedures were performed with the patients connected to cardiopulmonary bypass and their hearts arrested with cold blood cardioplegia $(\mathrm{n}=22)$ or induced fibrillation $(\mathrm{n}=2)$. Concomitant procedures included coronary artery bypass grafting in 18 cases $(9$ single, 6 double, 1 triple, and 2 quadruple bypasses), mitral valve reconstruction in 7 cases, aortic valve replacement in 2 cases, and use of intraaortic balloon pump in 2 cases. All procedures were performed in a similar fashion. The anterior wall was incised, and the transitional zone between infarcted and viable myocardium was visually identified. A Fontan purse-string suture was placed and tied, and the remaining opening was closed with overlapping felt-reenforced Prolene sutures either directly or with the use of a patch. The scar tissue was oversewn and excluded from the cavity. The exact amount of scar tissue or ventricle excluded from the cavity depended on the individual surgeon and was therefore based on personal judgment. All patients received the same routine intra- and postoperative care. Perioperatively, all patients received standard heart failure medication, including the administration of angiotensin-converting enzyme inhibitors or angiotensin II receptor blockers, beta-blockers, and diuretics.

\section{Cardiac Magnetic Resonance}

CMR was performed on a $1.5 \mathrm{~T}$ scanner (Intera; Philips, Best, The Netherlands) using an 8-element phased-array surface coil. Details of the volumetric, functional, and viability assessment have been described. ${ }^{2}$

\section{Determination of Cardiac Magnetic Resonance- Derived Volumetric, Geometric, Functional, and Viability Parameters}

Left ventricular volumes. All measurements were obtained from the horizontal long axis (4-chamber view), vertical long axis (2-chamber view), and a contiguous set of short-axis slices. The endo- and epicardial myocardial borders of the left ventricle were manually planimetered in all short-axis slices with a mouse-driven cursor using the software Extended MR Work Space (Release 2.6.1 2008; Philips Medical System, Nederland BV, The Netherlands); afterward, the software automatically multiplied these planimetered areas by the slice thickness. Papillary muscles were included in the LV cavity. Consequently, left ventricular volumes were summed to give the total ventricular volume at end-diastole and end-systole according to the Simpson rule algorithm. ${ }^{7}$ The left ventricular muscle mass was automatically generated by the software. For all volumetric parameters, the left ventricular end-diastolic and systolic volume indices were calculated using the formula by Du Bois and Du Bois. ${ }^{8}$

Left ventricular global and regional function. In addition to left ventricular ejection fraction and stroke volume, the cardiac index $\left(\mathrm{L} / \mathrm{min} \cdot \mathrm{m}^{2}\right)$ was calculated as the ratio of cardiac output and body surface area. ${ }^{7,9}$ Basal function (Figure 1,E) was determined using the following algorithm: The mean amount of equidistant slices of this acquisition depended on the length of the left ventricle and ranged from 9 to 12 (Figure 1, $C, D$ ). The volume for each slice was calculated from the slice area and slice thickness. The ratio of the mean end-diastolic and mean end-systolic volumes of the 3 most basal slices (blue markings in Figure $1, E$ ) was calculated and used to reflect regional basal EF. Regional basal EF therefore describes the fractional volume change during the cardiac cycle in the basal 3 slices.

Local wall motion was visually analyzed on the steady-state free-precession cine-magnetic resonance imaging by one experienced radiologist using the 17-segment model of the American Heart Association. ${ }^{10}$ Segments 17 to 13 represent the apical portion, segments 12 to 7 represent the mid-cavity, and segments 6 to 1 represent the basal portion of the ventricle. For each segment, the local wall motion was classified pre- and postoperatively using a qualitative scoring system with the following scale: $0=$ normokinetic, $1=$ hypokinetic, $2=$ akinetic, $3=$ dyskinetic.

To assess the completeness of revascularization from the bypass part of the procedure ( $75 \%$ of patients received at least 1 coronary artery bypass graft), we obtained information about the respective coronary circulation dominance and the localization and extension of the coronary artery stenoses. We matched these findings to the position of the bypass grafts. This matching allowed us to estimate with relatively high precision if a certain segment of the 17-segment model was completely revascularized or not. The assignment of segments to coronary artery territories was based on the recommendations of the American Heart Association, ${ }^{10}$ considering the limitation that there is considerable variability in myocardial blood supply.

\section{Geometric Indices}

Three different geometry indices, 2 already known from echocardiography, ${ }^{5}$ were used to describe left ventricular shape conditions pre- and postSVR by using patient-specific ventricular dimensions and volumes (Figure 1, $A-D$ ). The long-axis length (LAL) was defined as the distance from the apex to the center of the mitral valve (visualized by a reference line imposed at the level of the mitral annulus). The short-axis length (SAL) was defined as the distance between the interventricular septum and the lateral wall of the left ventricle perpendicular to and at the center of the LAL. 


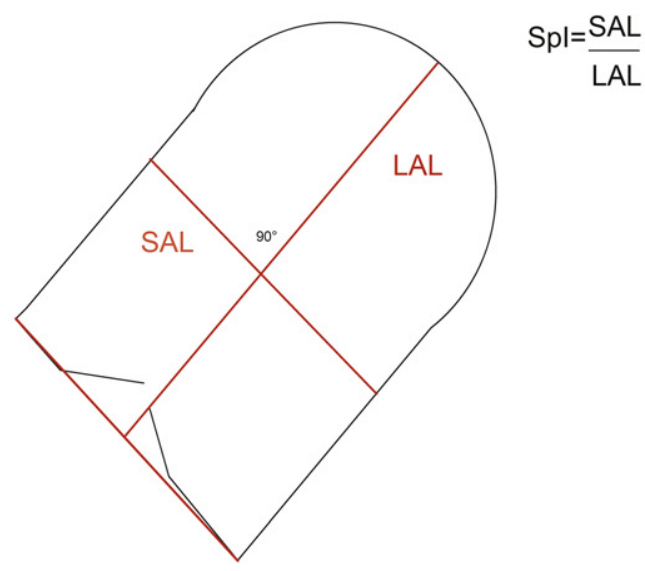

A

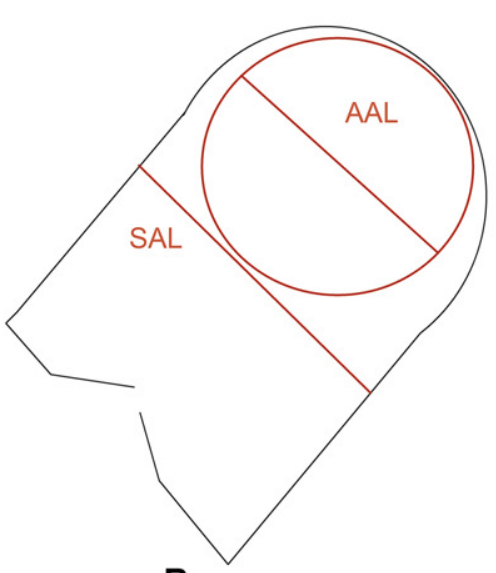

$\mathrm{ACl}=\frac{\mathrm{AAL}}{\mathrm{SAL}}$

B

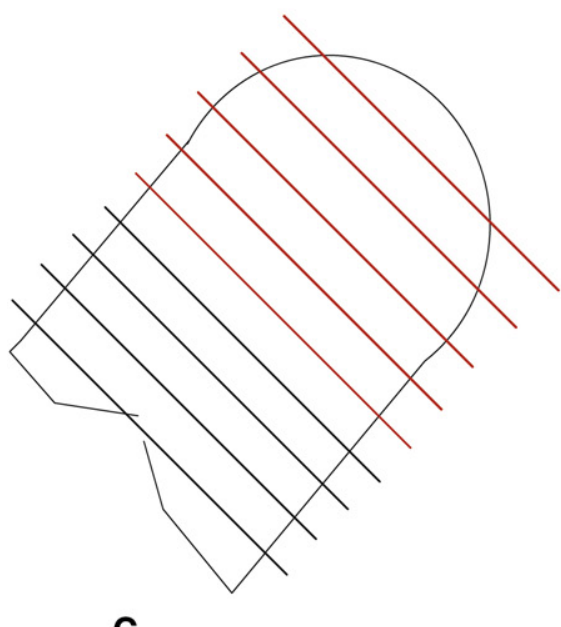

C
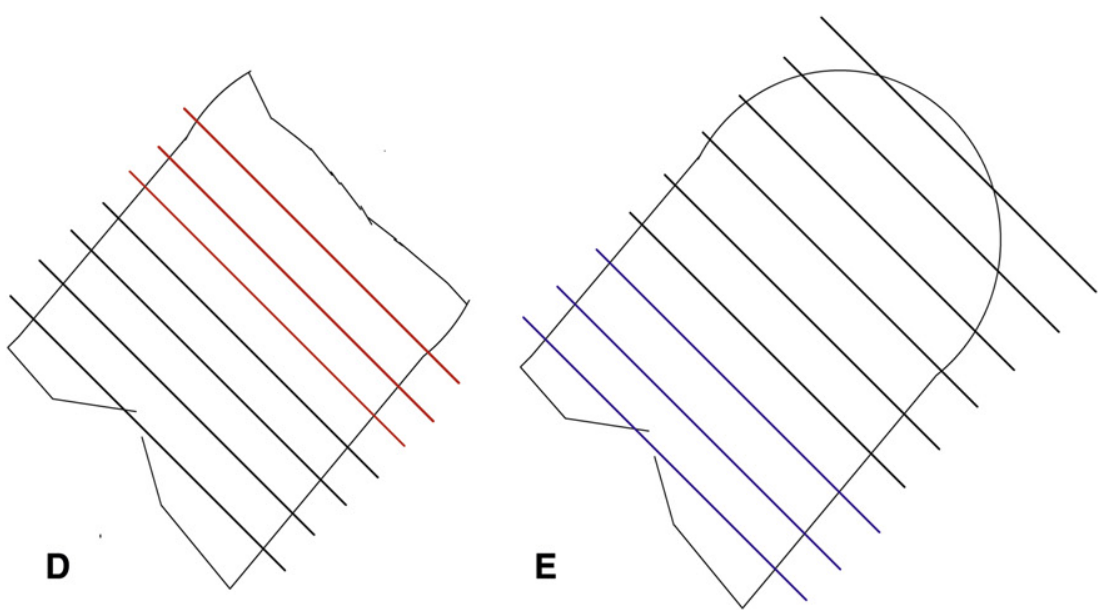

FIGURE 1. Determination of the examined geometry and functional indices. A, SpI is determined as the ratio of the internal diameters in SAL and LAL. B, ACI is determined as the ratio of the internal diameters in AAL and SAL. C, AVI is determined by the volume in the apical half of equidistant slices applied to the preoperative ventricle. D, For determination of the postoperative AVI, the volume in the same number of basal slices and the volume in the remaining apical slices are calculated and divided by each other (apical/basal). E, Regional basal EF is determined as the ratio of the mean end-diastolic and the mean end-systolic volume of the 3 most basal slices. SAL, Short-axis length; $L A L$, long-axis length; $A A L$, apical axi length; $A C I$, apical conicity index; $S p I$, sphericity index.

\section{Sphericity Index}

The sphericity index $(\mathrm{SpI})$ was defined as the ratio of the diameters SAL and LAL (Figure 1, A).

\section{Apical Conicity Index}

The apical conicity index (ACI) was obtained as described by Di Donato and colleagues. ${ }^{5}$ A sphere was fitted to the apex, and its diameter was then divided by the SAL (Figure 1, B).

\section{Apical Volume Index}

Figure 1, $C$ and $D$, illustrates how the apical volume index (AVI) was determined. The left ventricular volume was sectioned into short-axis slices of equal thickness starting at the mitral valve annulus during end-diastole and end-systole. The preoperative AVI was determined as the ratio of the volume represented by the apical half of the slices divided by the volume in the basal half of the slices. Postoperatively, the same slices were applied to the reconstructed ventricle starting at the mitral valve annulus during end-diastole and end-systole. The basal volume was again calculated from the same number of basal slices. Because of the reconstruction procedure, the number of apical slices was then reduced. Because the apical volume is likely to be reduced more than the volume in the basal sections by the operative procedure, the ratio is expected to decrease. However, the degree of decrease will depend on the amount of volume reduction and the amount of reshaping of the basal part. A spherical shape is likely to leave the basal volume unaffected, whereas the recreation of a conical shape is likely to slim the basal part.

\section{Scar Tissue Assessment}

The late gadolinium-DTPA enhancement images were analyzed, and the hyperenhancement area was quantified by planimetry in all acquired inversion recovery-gradient recalled echo short-axis slices using the same software by Philips (the section "Left ventricular volumes"). 
TABLE 1. Demographic data of the 24 patients having undergone surgical ventricular reconstruction and the division into subgroups by ejection fraction improvement

\begin{tabular}{lcccr}
\hline \multicolumn{1}{c}{ Parameters } & $\begin{array}{c}\text { All } \\
\text { patients }\end{array}$ & $\begin{array}{c}\boldsymbol{\Delta} \mathbf{E F} \\
>\mathbf{1 2} \%\end{array}$ & $\begin{array}{r}\boldsymbol{\Delta} \mathbf{E F} \\
<\mathbf{1 2} \%\end{array}$ & $\begin{array}{r}\boldsymbol{P} \\
\text { value }\end{array}$ \\
\hline Age, y & 58.7 & 61.7 & 57.1 & .354 \\
NYHA class & & & & \\
$\quad$ I & $4 / 24$ & $2 / 10$ & $2 / 14$ & 1.000 \\
II & $2 / 24$ & $1 / 10$ & $1 / 14$ & 1.000 \\
III & $11 / 24$ & $5 / 10$ & $6 / 14$ & 1.000 \\
IV & $7 / 24$ & $2 / 10$ & $5 / 14$ & 1.000 \\
Hypertension & $16 / 24$ & $6 / 10$ & $9 / 14$ & 1.000 \\
Diabetes & $9 / 24$ & $5 / 10$ & $4 / 14$ & .403 \\
Pulmonary hypertension & $4 / 24$ & $3 / 10$ & $1 / 14$ & .272 \\
PVD & $1 / 24$ & $0 / 10$ & $1 / 14$ & 1.000 \\
COPD & $4 / 24$ & $1 / 10$ & $3 / 14$ & .615 \\
Hyperlipidemia & $16 / 24$ & $6 / 10$ & $10 / 14$ & .673 \\
Previous PCI & $7 / 24$ & $2 / 10$ & $5 / 14$ & .653 \\
Prior heart operation & $2 / 24$ & $1 / 10$ & $1 / 14$ & 1.000 \\
Preoperative MI & $15 / 24$ & $5 / 10$ & $10 / 14$ & .403 \\
Coronary artery diseases & & & & \\
$\quad$ 1 vessel & $6 / 24$ & $3 / 10$ & $3 / 14$ & .665 \\
2 vessel & $7 / 24$ & $3 / 10$ & $4 / 14$ & 1.000 \\
3 vessel & $11 / 24$ & $4 / 10$ & $7 / 14$ & .697 \\
\hline P
\end{tabular}

$P$ values reflect comparison of the 2 subgroups. $P V D$, Peripheral vascular disease; COPD, chronic obstructive pulmonary disease; NYHA, New York Heart Association; $M I$, myocardial infarction; $P C I$, percutaneous coronary intervention.

Hyperenhancement was defined as scar tissue with a signal intensity of at least 2 standard deviations above the mean signal intensity of viable remote myocardium, ${ }^{2,11}$ in accordance with the typical morphologic aspect of infarcted myocardium. Finally, the planimetered scar areas were multiplied by the slice thickness and the total scar-mass was obtained and put into relation to the total myocardial mass. In addition, for each segment of the 17-segment model the local extension of the myocardial scar tissue was classified as transmural (at least $75 \%$ of the wall), intermediate $(50 \%)$, subendocardial $(<25 \%)$, or no infarction $(0 \%)$.

\section{Statistical Analysis}

Data are presented as mean \pm standard error of the mean. The 24 patients were subdivided into 2 groups according to their functional recovery (1 group with above average recovery of function and 1 group with below average recovery of function) to find predictors of functional outcome (see "Results"). Statistical analysis was performed using SPSS software 15.0 for windows, version 15.0.1 (SPSS Inc, Chicago, Ill). A paired, 2-tailed Student $t$ test, chi-square test, and Kaplan-Meier survival curve were used. We also performed a multivariate analysis to identify independent prognostic preoperative factors.

\section{RESULTS \\ Demographics}

Table 1 shows the demographic data of all patients. Patients were aged an average of 59 years and mainly in New York Heart Association class III or IV at the time of operation. The decision to perform SVR in our patients was mainly based on the presence of an apical aneurysm after myocardial infarction. As will be described below in further detail, ejection fraction improved on average by $12 \%$
TABLE 2. Operative parameters of the 24 patients and the division into subgroups by ejection fraction improvement

\begin{tabular}{|c|c|c|c|c|}
\hline Parameters & $\begin{array}{c}\text { All } \\
\text { patients }\end{array}$ & $\begin{array}{c}\Delta \mathbf{E F} \\
>12 \%\end{array}$ & $\begin{array}{r}\Delta \mathbf{E F} \\
<\mathbf{1 2} \%\end{array}$ & $\begin{array}{c}P \\
\text { value }\end{array}$ \\
\hline \multicolumn{5}{|l|}{ Urgency of operation } \\
\hline Elective & $14 / 24$ & $8 / 10$ & $6 / 14$ & .104 \\
\hline Urgent & $8 / 24$ & $2 / 10$ & $6 / 14$ & .388 \\
\hline Emergency & $2 / 24$ & $0 / 10$ & $2 / 14$ & .493 \\
\hline CABG & $18 / 24$ & $7 / 10$ & $11 / 14$ & .665 \\
\hline None & $6 / 24$ & $3 / 10$ & $3 / 14$ & .665 \\
\hline Single & $9 / 24$ & $4 / 10$ & $5 / 14$ & 1.000 \\
\hline Double & $6 / 24$ & $2 / 10$ & $4 / 14$ & 1.000 \\
\hline Triple & $1 / 24$ & $0 / 10$ & $1 / 14$ & 1.000 \\
\hline Quadruple & $2 / 24$ & $1 / 10$ & $1 / 14$ & 1.000 \\
\hline Concomitant MVR & $7 / 24$ & $5 / 10$ & $2 / 14$ & .085 \\
\hline Duration of operation (min) & 200 & 206 & 195 & .642 \\
\hline CPB (min) & 103 & 109 & 98 & .513 \\
\hline Aortic crossclamp time (min) & 58 & 57 & 58 & .933 \\
\hline \multicolumn{5}{|c|}{ Preoperative inotrope requirements } \\
\hline None & $20 / 24$ & $8 / 10$ & $12 / 14$ & 1.000 \\
\hline Low-dose & $4 / 24$ & $2 / 10$ & $2 / 14$ & 1.000 \\
\hline High-dose & $0 / 24$ & $0 / 10$ & $0 / 10$ & \\
\hline \multicolumn{5}{|c|}{ Postoperative inotrope requirements } \\
\hline None & $11 / 24$ & $4 / 10$ & $7 / 14$ & 697 \\
\hline Low-dose & $11 / 24$ & $6 / 10$ & $5 / 14$ & .408 \\
\hline High-dose & $2 / 24$ & $0 / 10$ & $2 / 14$ & .493 \\
\hline IABP & $2 / 24$ & $0 / 10$ & $2 / 14$ & 1.000 \\
\hline
\end{tabular}

$P$ values reflect comparison of the 2 subgroups. $C P B$, Cardiopulmonary bypass; IABP, intraaortic balloon pump; $M V R$, mitral valve reconstruction; $C A B G$, coronary artery bypass graft.

through the surgical procedure. By aiming to identify those patients who benefited most from the procedure, we divided the 24 patients into 2 groups: those with greater than $12 \%$ improvement in $\mathrm{EF}$ and those with less than $12 \%$ improvement in EF (Table 1). There were no significant demographic differences between the 2 groups.

\section{Operative Characteristics}

Table 2 shows the operative parameters of the patients. The majority of patients underwent the procedure electively. Two patients received an intraaortic balloon pump. Both of them were in the subgroup with poor improvement of function. The majority of patients also received bypass grafting, and one quarter of patients obtained a mitral valve repair for moderate to severe mitral regurgitation. Also, there were no statistically significant differences in the operative characteristics between the 2 subgroups.

The average degree of mitral regurgitation of patients who did not receive mitral valve reconstruction was grade $0.47 \pm 0.51$ before surgery and grade $0.41 \pm 0.51$ after surgery $(P=.668)$. Four patients improved, 2 patients worsened by 1 degree without the need for reintervention, and 15 patients remained the same. All reconstructed valves had more than mild mitral regurgitation before surgery and were competent at the end of the procedures $(n=7)$. New 
TABLE 3. Cardiac magnetic resonance-derived parameters of the 24 patients before and after surgical ventricular reconstruction

\begin{tabular}{lrrr}
\hline \multicolumn{1}{c}{ Parameters } & \multicolumn{1}{c}{ Pre } & \multicolumn{1}{c}{ Post } & P value \\
\hline LVEF $(\%)$ & $25 \pm 1.64$ & $37 \pm 2.28$ & $<.001$ \\
LVEDVI $\left(\mathrm{mL} / \mathrm{m}^{2}\right)$ & $148 \pm 10.8$ & $95 \pm 8.77$ & $<.001$ \\
LVESVI $\left(\mathrm{mL} / \mathrm{m}^{2}\right)$ & $114 \pm 10.9$ & $64 \pm 8.68$ & $<.001$ \\
LV-MM $(\mathrm{mL})$ & $180 \pm 12.8$ & $142 \pm 9.13$ & $<.001$ \\
CI $\left(\mathrm{L} / \mathrm{min} \cdot \mathrm{m}^{2}\right)$ & $2.49 \pm 0.13$ & $2.48 \pm 0.13$ & .961 \\
Apical motion & $2.80 \pm 0.07$ & $2.44 \pm 0.13$ & .007 \\
Basal motion & $0.76 \pm 0.08$ & $0.56 \pm 0.08$ & .001 \\
RBEF $(\%)$ & $52.9 \pm 3.41$ & $54.5 \pm 3.30$ & .530 \\
SpI $(4 \mathrm{CH}-$ dia) & $0.60 \pm 0.01$ & $0.76 \pm 0.03$ & $<.001$ \\
ACI $(4 \mathrm{CH}-\mathrm{dia})$ & $0.71 \pm 0.03$ & $0.58 \pm 0.02$ & $<.001$ \\
AVI & & & \\
$\quad$ Diastole & $0.45 \pm 0.02$ & $0.26 \pm 0.02$ & $<.001$ \\
$\quad$ Systole & $0.54 \pm 0.02$ & $0.32 \pm 0.03$ & $<.001$ \\
Total scar (mL) & $42.98 \pm 5.56$ & n.a. & \\
Total scar $(\%)$ & $24.56 \pm 2.88$ & n.a. & \\
Apical scar $(\%)$ & $71.67 \pm 1.58$ & n.a. & \\
Mid-cavity scar $(\%)$ & $37.29 \pm 3.22$ & n.a. & \\
Basal scar $(\%)$ & $10 \pm 2.53$ & n.a. & \\
\hline
\end{tabular}

Basal and apical motion scale: $3=$ dyskinesia, $2=$ akinesia, $1=$ hypokinesia, $0=$ normokinesia. $L V E F$, Left ventricular ejection fraction; $L V E D V I$, left ventricular end-diastolic volume index; LVESVI, left ventricular end-systolic volume index; $L V-M M$, left ventricular muscle mass; $C I$, cardiac index; $R B E F$, regional basal ejection fraction.

York Heart Association functional class improved in all patients on average from $2.9 \pm 1.0$ to $2.1 \pm 1.2(P=.009)$.

\section{Cardiac Magnetic Resonance Assessment of Function, Volumes, and Geometry}

Table 3 shows the CMR data of the patients before and after SVR. Consistent with other reports, ${ }^{4,12}$ our study found a significant reduction in left ventricular volumes associated with the already mentioned improvement in ejection fraction by $12 \%$. The SpI was increased by SVR, indicating the creation of ball shapes by the procedure. The ACI and AVI decreased, which is consistent with aneurysm removal. The preoperative ventricular muscle mass consisted to one quarter of the scar. Its relative proportion was greatest in the apical and lowest in the basal region.

Figure 2 illustrates the change of left ventricular ejection fraction of all patients. The average increase was $12 \%$. On the basis of these outcomes, we divided the group into 2 subgroups described above. Table 4 shows the CMR-derived parameters for the 2 subgroups. There was no difference in volume reductions, global amount of scar, or sphericity or apical conicity indices determined. There was also no difference in the regional basal EF, indicating that the degree of basal wall motion may not be predictive for postoperative function. Table E1 shows the local scar tissue distribution according to the American Heart Association 17-segment model, which was used as

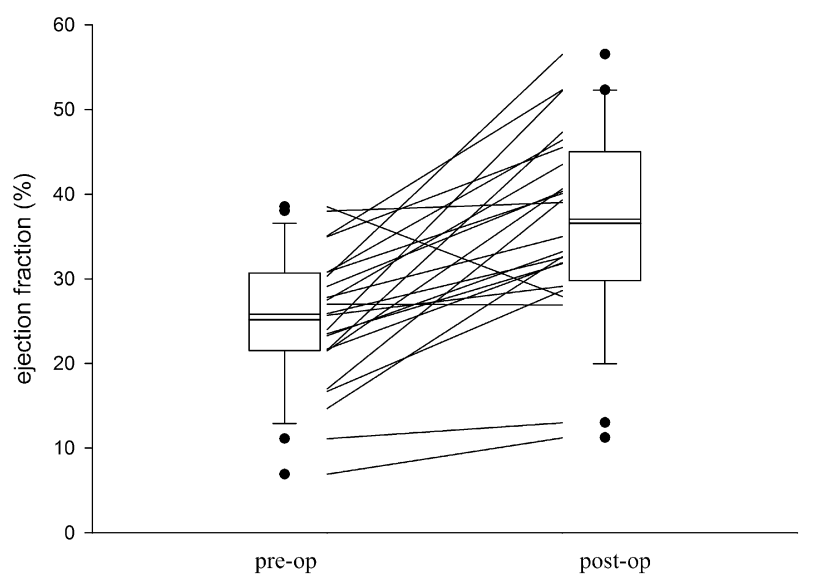

FIGURE 2. EF (\%) of all patients before and after SVR and their individual direction of change.

the basis for our apical, mid-cavity, and basal region assessment (see "Materials and Methods" for details). The scar tissue distribution was mostly transmural in the apical, intermediate in the mid-cavity, and subendocardial in the basal region. There was no difference between the 2 subgroups in the apical and mid-cavity region. Because of this finding, we postulated that differences in the outcome of this homogenous cohort should be reflected in the basal scar distribution, because the residual contractility of the ventricle is generated in this area and should be affected by scar tissue.

Figure 3, A, illustrates the fractional scar tissue in the basal myocardium. Patients with poor improvement of postoperative EF had more basal scar than those with large EF improvement. Figure 3, $B$, shows the newly introduced apical volume index. Patients with large EF improvements had smaller AVI after SVR than those with lower improvements, indicating that greater volume reductions during surgery are associated with better functional outcome.

Table E2 shows the local wall motion analysis on the basis of the 17-segment model of the American Heart Association and on our qualitative scoring system. The scoring system indicated significant improvements in function in the mid-cavity and basal regions. On the basis of our assessment of complete revascularization (see "Materials and Methods" for details), we distinguished segments that were revascularized from those that were not revascularized. According to this approximation, only $22 \%$ of all improvements of regional function were located in segments that have received revascularization and $77 \%$ of all regional functional improvements were located in non-revascularized segments. Functional improvements in the basal regions were associated with the greatest lack of revascularization $(86 \%)$, indicating that the functional improvement may be more influenced by the SVR procedure than by revascularization. 
TABLE 4. Comparison of examined volumes, functional, geometric, and viability parameters between the 2 subgroups divided by ejection fraction improvement

\begin{tabular}{|c|c|c|c|c|}
\hline \multirow[b]{2}{*}{ Parameters } & \multicolumn{2}{|c|}{$\Delta \mathrm{EF}>12 \%$} & \multicolumn{2}{|c|}{$\Delta \mathrm{EF}<12 \%$} \\
\hline & Preoperative & Postoperative & Preoperative & Postoperative \\
\hline LVEF & $24 \pm 2.17$ & $44 \pm 2.80 *$ & $26 \pm 2.39$ & $31 \pm 2.50 *$ \\
\hline LVEDVI $\left(\mathrm{mL} / \mathrm{m}^{2}\right)$ & $146 \pm 14.7$ & $81 \pm 10.2 *$ & $150 \pm 15.7$ & $106 \pm 12.7 *$ \\
\hline LVESVI $\left(\mathrm{mL} / \mathrm{m}^{2}\right)$ & $113 \pm 13.2$ & $47 \pm 8.41^{*}$ & $115 \pm 16.7$ & $77 \pm 12.9^{*}$ \\
\hline RBEF (\%) & $54 \pm 4.88$ & $58 \pm 4.48^{*}$ & $53 \pm 4.85$ & $52 \pm 4.70 *$ \\
\hline Basal wall motion & $0.70 \pm 0.13$ & $0.45 \pm 0.11$ & $0.80 \pm 0.11$ & $0.64 \pm 0.10^{*}$ \\
\hline SpI & $0.57 \pm 0.02$ & $0.77 \pm 0.05$ & $0.61 \pm 0.02$ & $0.75 \pm 0.03$ \\
\hline ACI & $0.71 \pm 0.05$ & $0.56 \pm 0.04^{*}$ & $0.71 \pm 0.03$ & $0.60 \pm 0.02$ \\
\hline Total scar (mL) & $41 \pm 10.4$ & n.a. & $44 \pm 6.29$ & n.a. \\
\hline Total scar $(\%)$ & $21 \pm 5$ & n.a. & $26 \pm 4$ & n.a. \\
\hline Apical scar $(\%)$ & $74 \pm 1$ & n.a. & $70 \pm 3$ & n.a. \\
\hline Mid-cavity scar (\%) & $39 \pm 5$ & n.a. & $38 \pm 4$ & n.a. \\
\hline Basal scar (\%) & $03 \pm 2$ & n.a. & $15 \pm 4 \dagger$ & n.a. \\
\hline
\end{tabular}

Basal wall motion scale: $3=$ dyskinesia, $2=$ akinesia, $1=$ hypokinesia, $0=$ normokinesia. $L V E F$, Left ventricular ejection fraction; $L V E D V I$, left ventricular end-diastolic volume index; $L V E S V I$, left ventricular end-systolic volume index; $R B E F$, regional basal ejection fraction. ${ }^{*} P<.05$ versus preoperative. $\dagger P<.05 \mathrm{vs} \Delta \mathrm{EF}>12 \%$.

\section{Midterm Survival}

Figure 4 shows a Kaplan-Meier survival curve for the 2 subgroups. In our cohort, the mean survival time was 66 months. In this time, 2 patients $(8.3 \%)$ died 3 and 13 months after surgery. Both showed preoperatively a high amount of myocardial scar (27.6\% and $46.7 \%)$. Of note, both had a below average improvement in ejection fraction postoperatively $(4.3 \%$ and $8.3 \%)$.

\section{DISCUSSION}

We demonstrated in this analysis that the assessment of regional scar content, especially in the basal region of the heart may help to determine a subgroup of patients who potentially benefits from surgical ventricular reconstruction (SVR). We also showed that the previously described geometric indices and shape beliefs are not associated with an improvement in postoperative function. Finally, we showed that the degree of volume reducing reflected in the newly introduced apical volume index (AVI) pre and post-surgery appeared to have a global functional impact. However, multivariate analysis failed to identify the above mentioned parameters as independent prognostic factors. These findings need further discussion.

Although the study is to date the largest one presenting pre- and postoperative CMR in patients having received SVR, the total number of 24 patients is too small to expect a multivariate analysis to deliver final results. We therefore arrived at our conclusions with the understanding that our results are hypothesis generating. However, they are currently of great interest because of the overall negative results of the SVR hypothesis of the STICH trial. ${ }^{4}$ This trial demonstrated no added benefit of SVR to conventional bypass surgery alone. However, because of the lack of specific inclusion criteria for SVR, the trial's patient population having received SVR is heterogeneous and contains patients with dilated hearts and mainly akinetic anterior walls, and patients with clearly defined apical aneurysms of different sizes and often adequately maintained contractility in the remaining ventricle. Therefore, there should be a subgroup (those with an improvement of left ventricular function above the mean) that benefits from the procedure. However, it is important to realize in this context that for every patient who benefits, the entire cohort contains a counterpart who takes harm. Therefore, identifying preoperative determinants of outcome is extremely important.

Intuitively, one would assume that function in the remaining myocardium might be a predictor of outcome. However, our data indicate that mid-cavity and basal contractility do not correlate with postoperative global function. A most recent report from the STICH trial supports this finding by being unable to identify subgroups of patients receiving benefit or harm from SVR based on regional contractility. ${ }^{13}$ However, regional scar content in the basal part of the left ventricle was inversely correlated with postoperative function in our analysis. These results are consistent with those of Takeda and colleagues, ${ }^{14}$ who assessed regional scar content in a smaller series than ours and did not assess global scar content and the geometric indices. Nevertheless, the evidence for basal scar content to be a preoperative predictor of outcome is growing, ${ }^{14,15}$ and larger studies are required to assess its true prognostic impact. This discrepancy between basal contractility and basal scar content as a predictor of functional outcome reflects the methodical superiority of late gadolinium-DTPA enhancement to a qualitative scoring system of wall motion only. The advantage of late gadolinium-DTPA enhancement may be that it objectively assesses irreversible myocardial damage, whereas wall motion changes may vary significantly and are influenced by dynamic factors (eg, volume load). ${ }^{16}$ 

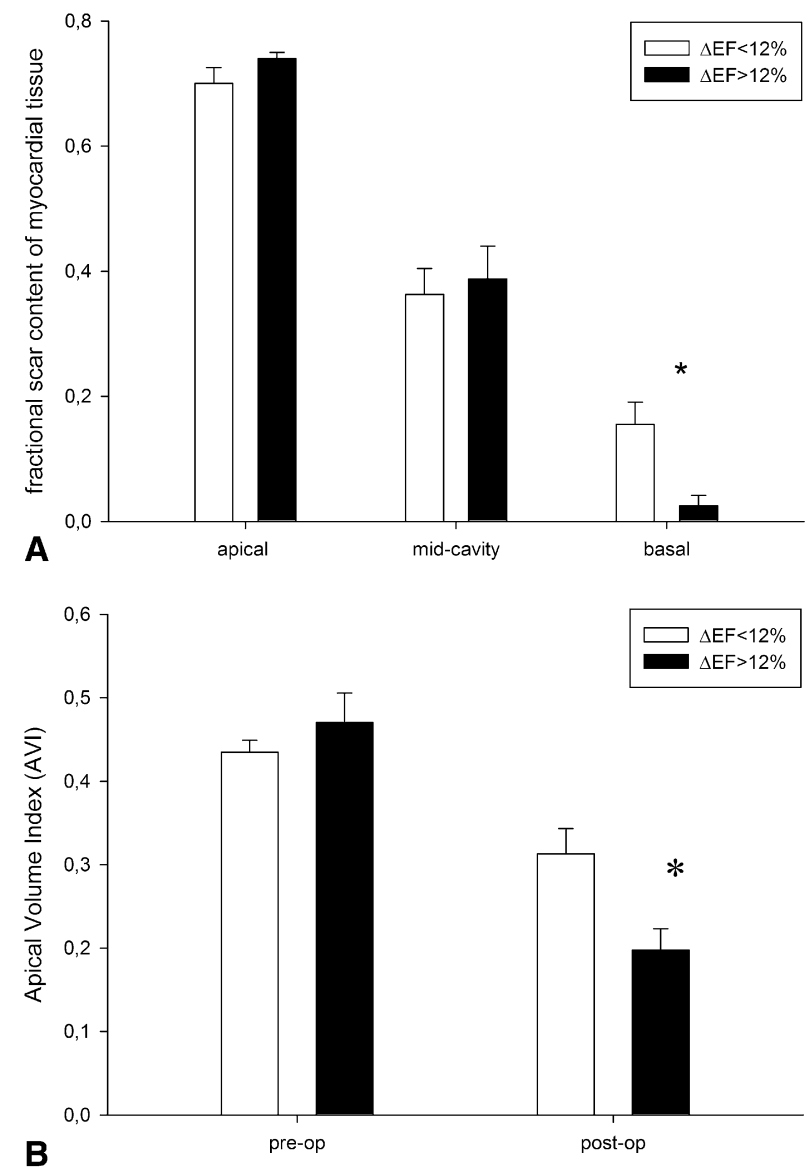

FIGURE 3. A, Fractional scar content of myocardial scar tissue in the apical, mid-cavity, and basal regions illustrated for the subgroups of patients with $\Delta \mathrm{EF}<12 \%$ (open bars) and $\Delta \mathrm{EF}>12 \%$ (filled bars). B, AVI before and after SVR in the subgroups of patients with $\triangle \mathrm{EF}<12 \%$ (open bars) and $\Delta \mathrm{EF}>12 \%$ (filled bars) showing the significant difference between the groups. A, B, Note the significantly smaller amount of basal scar and the significantly greater reduction in AVI in patients with better functional improvement. $A V I$, Apical volume index; $E F$, ejection fraction.

Another issue that has raised heated debates is the shape of the pre- and postoperative ventricle. Buckberg and colleagues ${ }^{17}$ and Menicanti and Di Donato ${ }^{18}$ reported that the creation of a cone-shaped ventricle is important, and specific modifications in the conduct of this procedure aiming at that shape have been described. ${ }^{19,20}$ However, there is currently no evidence that a difference in shape affects outcome. To the contrary, the surgeon reporting the best result in this group of patients (V. Dor) has the highest propensity to create ball-shaped ventricles (because of his volume assessment with a ball-shaped device and implantation of mainly round patches). ${ }^{21}$ Our analysis of the recently introduced geometric indices addressing shape (signal intensity, ACI) supports this notion. There seems to be no relationship between the change in shape according to the $\mathrm{SpI}$ and ACI and the degree in functional improvement after

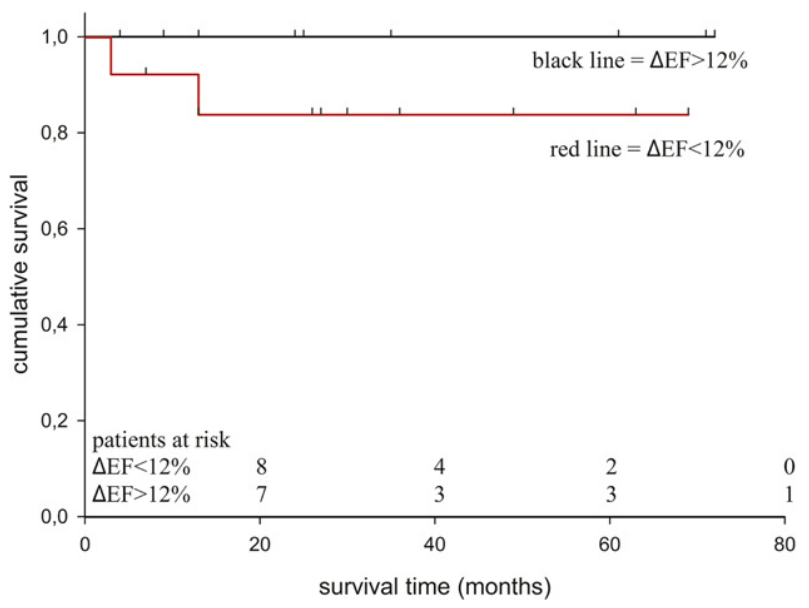

FIGURE 4. Kaplan-Meier survival curve for the 2 examined subgroups $(\Delta \mathrm{EF}<12 \%$ red line and $\Delta \mathrm{EF}>12 \%$ black line $) . E F$, Ejection fraction.

surgery. Although it seems from our data that ball shapes do not eliminate the possibility of significant functional improvement, we cannot make any statement about the impact of conical shapes because most of our patients had more ball-type shapes. However, because of the good functional improvements and the tendency to spherical ventricular shapes post-SVR in our cohort, we conclude that spherical shape is at least not absolutely contradicting good functional outcome.

We introduced a new volume index with this study. The preoperative AVI is determined as the ratio of the volume represented by the apical half divided by the volume of the basal half of the ventricle. They have been determined by sectioning the ventricle in equidistant slices and determining a basal set of slices and an apical set of slices. Because of the apical localization of the procedure, the apical set of slices was reduced and not the basal set of slices. Thus, the significant reduction of the volume indices postoperatively was expected. However, the statistically larger AVI reduction in patients with good EF improvement was not predictable. One may argue that more aggressive volume reduction may result in better functional outcome. However, regional contractility was not different between the 2 groups, and global EF is a function of ventricular volumes. Thus, the association of smaller volumes with larger global postoperative ejection fractions seems to be a mathematic rather than a functional effect. In addition to this functional considerations, it is always difficult to distinguish the effects of the SVR procedure from the effects of revascularization. Despite this limitation, we do provide evidence in this article that SVR seems to have an effect on contractile function. This conclusion is based on our matching of regional contractility to the presence or the lack of concomitant revascularization in the 17 segments. Because most of the segments experienced functional improvement but only one fifth received revascularization through bypass grafting, 
it is reasonable to conclude that SVR has a functional impact independent of revascularization.

The above reasoning on the interplay of volume, volume reduction, and ejection fraction also reflects the greatest limitation in this study (ie, the limited number of patients as already eluded to before). Because of this small number, we were not able to assess the impact of the procedure on clinical outcome independently of function. Thus, the question whether the differences in functional outcome will help to identify the expected subgroup of patients deriving a survival benefit cannot be fully answered. Our survival curve may serve as a small and indirect token of support for this connection (both patients who died were in the group with poor functional recovery), but certainly not as proof. Despite these limitations, our analysis is still the largest currently available one, and the evidence we provide strongly suggests that the assessment of regional parameters (specifically basal scar content and the newly introduced apical volume index) deserve further assessment in larger trials with preoperative primary outcome parameters.

\section{CONCLUSIONS}

The assessment of regional scar content may help to determine a subgroup of patients who benefit from surgical ventricular reconstruction. The previously described geometric indices and shape beliefs are not associated with improvement in postoperative function. Because these conclusions are not backed by the results of the multivariate analysis, there is a great need for a larger study to support these results.

\section{References}

1. Kim RJ, Wu E, Rafael A, Chen EL, Parker MA, Simonetti O, et al. The use of contrast-enhanced magnetic resonance imaging to identify reversible myocardial dysfunction. N Engl J Med. 2000;343:1445-53.

2. Gutberlet M, Frohlich M, Mehl S, Amthauer H, Hausmann H, Meyer R, et al. Myocardial viability assessment in patients with highly impaired left ventricular function: comparison of delayed enhancement, dobutamine stress MRI, enddiastolic wall thickness, and TI201-SPECT with functional recovery after revascularization. Eur Radiol. 2005;15:872-80.

3. Dor V, Sabatier M, Di Donato M, Montiglio F, Toso A, Maioli M. Efficacy of endoventricular patch plasty in large postinfarction akinetic scar and severe left ventricular dysfunction: comparison with a series of large dyskinetic scars. J Thorac Cardiovasc Surg. 1998;116:50-9.

4. Jones RH, Velazquez EJ, Michler RE, Sopko G, Oh JK, O'Connor CM, et al. Coronary bypass surgery with or without surgical ventricular reconstruction. $N$ Engl J Med. 2009;360:1705-17.

5. Di Donato M, Dabic P, Castelvecchio S, Santambrogio C, Brankovic J, Collarini L, et al. Left ventricular geometry in normal and post-anterior myocardial infarction patients: sphericity index and 'new' conicity index comparisons. Eur J Cardiothorac Surg. 2006;29(Suppl 1):S225-30.

6. Maffessanti F, Corsi C, Lang RM, Caiani EG. Development of a method for left ventricular shape evaluation based on surfaces obtained by real-time 3D echocardiographic images. Comput Cardiol. 2007;34:1-4.

7. Folland ED, Parisi AF, Moynihan PF, Jones DR, Feldman CL, Tow DE. Assessment of left ventricular ejection fraction and volumes by real-time, two-dimensional echocardiography. A comparison of cineangiographic and radionuclide techniques. Circulation. 1979;60:760-6.

8. Du Bois D, Du Bois EF. A formula to estimate the approximate surface area if height and weight be known. 1916. Nutrition. 1989;5:303-13.

9. Gutberlet M, Spors B, Thoma T, Bertram H, Denecke T, Felix R, et al. Suspected chronic myocarditis at cardiac MR: diagnostic accuracy and association with immunohistologically detected inflammation and viral persistence. Radiology. 2008;246:401-9.

10. Cerqueira MD, Weissman NJ, Dilsizian V, Jacobs AK, Kaul S, Laskey WK, et al. Standardized myocardial segmentation and nomenclature for tomographic imaging of the heart. A statement for healthcare professionals from the Cardiac Imaging Committee of the Council on Clinical Cardiology of the American Heart Association. Int J Cardiovasc Imaging. 2002;18:539-42.

11. Lauerma K, Niemi P, Hanninen H, Janatuinen T, Voipio-Pulkki LM, Knuuti J, et al. Multimodality MR imaging assessment of myocardial viability: combination of first-pass and late contrast enhancement to wall motion dynamics and comparison with FDG PET-initial experience. Radiology. 2000;217: 729-36.

12. Athanasuleas CL, Buckberg GD, Stanley AW, Siler W, Dor V, DiDonato M, et al. Surgical ventricular restoration: the RESTORE Group experience. Heart Fail Rev. 2004;9:287-97.

13. Oh J, Menicanti L, Bochenek A, Pohost G, Cherniavsky A, Bonow R, Wos S, et al., on behalf of the STICH Investigators. Influence of Baseline Global and Regional Cardiac Function on Outcome of Patients Randomized to CABG With or Without Surgical Ventricular Reconstruction (SVR). Late Breaking Clinical Trial Update, American Heart Association, November 17, 2009.

14. Takeda K, Matsumiya G, Matsue H, Hamada S, Sakaki M, Sakaguchi T, et al. Use of quantitative analysis of remote myocardial fibrosis with delayed-enhancement magnetic resonance imaging to predict outcomes after surgical ventricular restoration for ischemic cardiomyopathy. J Thorac Cardiovasc Surg. 2008;136: 1514-21.

15. Ogawa M, Doi K, Yamada Y, Fukumoto A, Okawa K, Kan'bara T, et al. Surgical ventricular restoration based on evaluation of myocardial viability with delayedenhanced magnetic resonance imaging. Gen Thorac Cardiovasc Surg. 2007;55: 149-57.

16. Bombardini T. Myocardial contractility in the echo lab: molecular, cellular and pathophysiological basis. Cardiovasc Ultrasound. 2005;3:27.

17. Buckberg GD. Form versus disease: optimizing geometry during ventricular restoration. Eur J Cardiothorac Surg. 2006;29(Suppl 1):S238-44.

18. Menicanti L, Di Donato M. Surgical left ventricle reconstruction, pathophysiologic insights, results and expectation from the STICH trial. Eur J Cardiothorac Surg. 2004;26(Suppl 1):S42-7.

19. Di Donato M, Sabatier M, Dor V, Gensini GF, Toso A, Maioli M, et al. Effects of the Dor procedure on left ventricular dimension and shape and geometric correlates of mitral regurgitation one year after surgery. J Thorac Cardiovasc Surg. 2001;121:91-6.

20. Athanasuleas CL, Stanley AW, Buckberg GD, Dor V, Di Donato M, Siler W. Surgical anterior ventricular endocardial restoration (SAVER) for dilated ischemic cardiomyopathy. Semin Thorac Cardiovasc Surg. 2001;13:448-58.

21. Dor V, Sabatier M, Montiglio F, Civaia F, DiDonato M. Endoventricular patch reconstruction of ischemic failing ventricle. a single center with 20 years experience. advantages of magnetic resonance imaging assessment. Heart Fail Rev. 2004;9:269-86. 
TABLE E1. Fractional content of scar tissue in percentage of patients' left ventricles before surgical ventricular reconstruction according to 17 segment model (American Heart Association)

\begin{tabular}{lccc}
\hline Myocardial region & All patients & $\mathbf{\Delta E F}>\mathbf{1 2} \%$ & $\boldsymbol{\Delta E F}<\mathbf{1 2} \%$ \\
\hline Total scar (\%) & $25 \pm 3$ & $15 \pm 5$ & $14 \pm 4$ \\
Regional scar (\%) & & & \\
Apical & & & .338 \\
$\quad$ Apex (17) & $0.75 \pm 0.00$ & $0.75 \pm 0.00$ & $0.75 \pm 0.00$ \\
Lateral (16) & $0.69 \pm 0.03$ & $0.73 \pm 0.03$ & $0.66 \pm 0.06$ \\
Inferior (15) & $0.69 \pm 0.04$ & $0.73 \pm 0.03$ & $0.66 \pm 0.06$ \\
Septal (14) & $0.72 \pm 0.02$ & $0.75 \pm 0.00$ & $0.70 \pm 0.03$ \\
Anterior (13) & $0.73 \pm 0.01$ & $0.75 \pm 0.00$ & $0.73 \pm 0.02$ \\
Mid-cavity & & & .310 \\
Anterolateral (12) & $0.28 \pm 0.07$ & $0.28 \pm 0.11$ & .350 \\
Inferolateral (11) & $0.25 \pm 0.06$ & $0.23 \pm 0.10$ & $0.27 \pm 0.08$ \\
Inferior (10) & $0.24 \pm 0.06$ & $0.33 \pm 0.11$ & $0.18 \pm 0.05$ \\
Inferoseptal (9) & $0.38 \pm 0.06$ & $0.45 \pm 0.10$ & $0.36 \pm 0.09$ \\
Anteroseptal (8) & $0.55 \pm 0.04$ & $0.53 \pm 0.10$ & $0.61 \pm 0.04$ \\
$\quad$ Anterior (7) & $0.54 \pm 0.05$ & $0.53 \pm 0.10$ & .410 \\
Basal & & & .960 \\
Anterolateral (6) & $0.05 \pm 0.04$ & $0.00 \pm 0.00$ & .735 \\
Inferolateral (5) & $0.03 \pm 0.03$ & $0.00 \pm 0.00$ & .198 \\
Inferior (4) & $0.03 \pm 0.02$ & $0.00 \pm 0.00$ & .482 \\
Inferoseptal (3) & $0.01 \pm 0.04$ & $0.03 \pm 0.03$ & .413 \\
Anteroseptal (2) & $0.23 \pm 0.06$ & $0.10 \pm 0.06$ & .523 \\
Anterior (1) & $0.14 \pm 0.05$ & $0.03 \pm 0.03$ & $0.10 \pm 0.06$ \\
\hline
\end{tabular}

Transmural $>0.75$, intermediate 0.5 , subendocardial $<0.25$, no infarction 0 .

TABLE E2. Local wall motion assessed by cardiac magnetic resonance pre- and post- surgical ventricular reconstruction of all 24 patients according to the American Heart Association 17-segment model

\begin{tabular}{|c|c|c|c|}
\hline Myocardial region & Preoperative & Postoperative & $P$ value \\
\hline \multicolumn{4}{|l|}{ Apical } \\
\hline Apex (17) & $2.9 \pm 0.06$ & $2.75 \pm 0.11$ & .103 \\
\hline Lateral (16) & $2.75 \pm 0.11$ & $2.38 \pm 0.17$ & .026 \\
\hline Inferior (15) & $2.75 \pm 0.10$ & $2.21 \pm 0.17$ & .002 \\
\hline Septal (14) & $2.79 \pm 0.09$ & $2.33 \pm 0.18$ & .008 \\
\hline Anterior (13) & $2.79 \pm 0.09$ & $2.54 \pm 0.13$ & .031 \\
\hline \multicolumn{4}{|l|}{ Mid-cavity } \\
\hline Anterolateral (12) & $1.33 \pm 0.13$ & $0.63 \pm 0.15$ & $<.001$ \\
\hline Inferolateral (11) & $1.66 \pm 0.13$ & $1.13 \pm 0.15$ & $<.001$ \\
\hline Inferior (10) & $1.5 \pm 0.13$ & $1.00 \pm 0.14$ & .001 \\
\hline Inferoseptal (9) & $1.5 \pm 0.10$ & $1.13 \pm 0.10$ & $<.001$ \\
\hline Anteroseptal (8) & $1.5 \pm 0.10$ & $1.0 \pm 0.10$ & $<.001$ \\
\hline Anterior (7) & $1.33 \pm 0.16$ & $0.79 \pm 0.13$ & .001 \\
\hline \multicolumn{4}{|l|}{ Basal } \\
\hline Anterolateral (6) & $0.33 \pm 0.10$ & $0.25 \pm 0.09$ & .162 \\
\hline Inferolateral (5) & $0.33 \pm 0.10$ & $0.25 \pm 0.09$ & .162 \\
\hline Inferior (4) & $0.83 \pm 0.13$ & $0.63 \pm 0.12$ & .022 \\
\hline Inferoseptal (3) & $1.04 \pm 0.14$ & $0.79 \pm 0.12$ & .011 \\
\hline Anteroseptal (2) & $1.13 \pm 0.13$ & $0.83 \pm 0.12$ & .005 \\
\hline Anterior (1) & $0.92 \pm 0.12$ & $0.63 \pm 0.12$ & .005 \\
\hline
\end{tabular}

Wall motion scale: $3=$ dyskinesia, $2=$ akinesia, $1=$ hypokinesia, $0=$ normokinesia. 